\title{
A Madagascar Sponge Batzella sp. as a Source of Alkylated Iminosugars
}

\author{
[batzella_SI_v5; 10/26/04] \\ Nathaniel L. Segraves, ${ }^{\dagger}$ and Phillip Crews ${ }^{*}, \dagger$ \\ ${ }^{\dagger}$ Department of Chemistry and Biochemistry and Institute for Marine Sciences, \\ University of California, Santa Cruz, California 95064
}

\section{Supporting Information}

\section{Experimental Procedure}

Figure S1. Known Iminosugars of Microbial (M) and Plant (P) sources.

Figure S2. Iminosugars with the same molecular formula as bastzellaside A.

Figure S3. Plant-derived alkaloids considered related to batzellasides A-C.

Figure S4. Marine natural products related to batzellasides A-C.

Figure S5. ${ }^{1} \mathrm{H}$ NMR spectrum of batzellaside A (3), (500 MHz, MeOH- $\left.d_{4}\right)$.

Figure S6. ${ }^{13} \mathrm{C}$ NMR spectrum of batzellaside A (3), (125 MHz, MeOH-d $\left.{ }_{4}\right)$.

Figure S7. DEPT spectrum of batzellaside A (3), $\left(500 \mathrm{MHz}, \mathrm{MeOH}-d_{4}\right)$.

Figure S8. ${ }^{1} \mathrm{H}{ }^{1} \mathrm{H}$ COSY spectrum of batzellaside A (3), (500 MHz, MeOH-d $\left.d_{4}\right)$.

Figure S9. gHMQC spectrum of batzellaside A (3), (500 MHz, MeOH-d s. $_{4}$.

Figure S10. gHMBC ( $=8 \mathrm{~Hz})$ spectrum of batzellaside A (3), (500 MHz, MeOH$\left.d_{4}\right)$.

Figure S11. gHMBC ( $\mathrm{J}=4 \mathrm{~Hz})$ spectrum of batzellaside A (3), (500 MHz, MeOH$\left.d_{4}\right)$.

Figure S12. ${ }^{1} \mathrm{H}^{1} \mathrm{H}$ NOESY spectrum of batzellaside A (3), (500 MHz, MeOH-d $)$.

Figure S13. ${ }^{1} \mathrm{H}$ NMR spectrum of batzellaside B (4), $\left(500 \mathrm{MHz}, \mathrm{MeOH}-d_{4}\right)$.

Figure S14. ${ }^{1} \mathrm{H}$ NMR spectrum of batzellaside $\mathrm{C}(5),\left(500 \mathrm{MHz}, \mathrm{MeOH}-d_{4}\right)$.

Figure S15. Above Water Photograph of 00216.

Figure S16. Outline of isolation of halitoxin and batzellasides A - C. 
Figure S1. Known Iminosugars of Microbial (M) and Plant (P) sources.

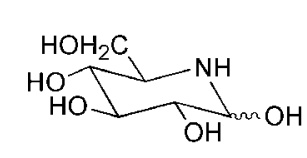

nojirimycin (1) M

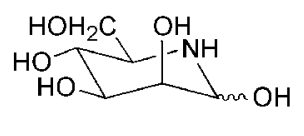

mannojirimycin (14) M

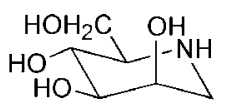

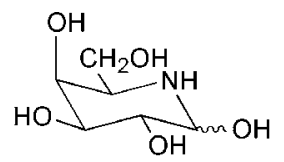

galactonojirimycin (15)

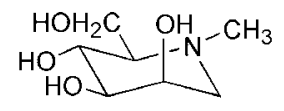

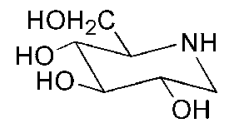

1-deoxynojirimycin (6) $\mathrm{M} / \mathrm{P}$

DNJ

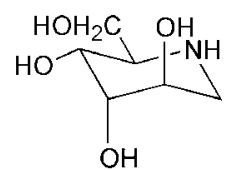

N-methyl-DNJ (16) P 1-deoxymannojirimycin (17) M/P N-methyl-DMJ (18) P 1-deoxyaltronojirimycin (19) P DMJ

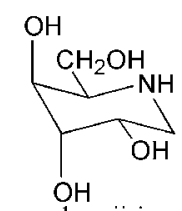

1-deoxygulonojirimycin (20) P 1,6-dideoxynojirimycin (21) P
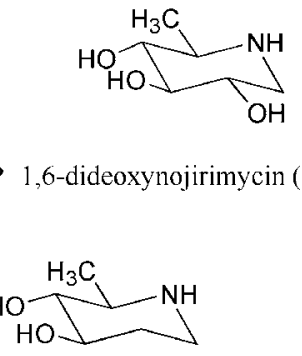

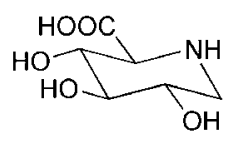

$\mathrm{OH}$

3,4-di-epi-fagomine (12) P 6-deoxyfagomine (23) P

2S-carboxy-3R,4R,5S-

trihydroxypiperidine (24) $\mathrm{P}$

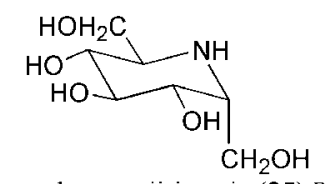

$\alpha$-homonojirimycin (25) P

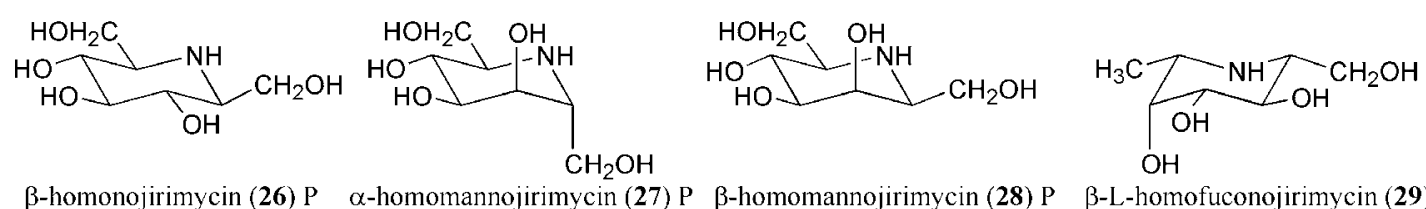

$\beta$-homonojirimycin (26) P $\quad \alpha$-homomannojirimycin (27) $\mathrm{P} \quad \beta$-homomannojirimycin (28) $\mathrm{P} \quad \beta$-L-homofuconojirimycin (29) $\mathrm{P}$
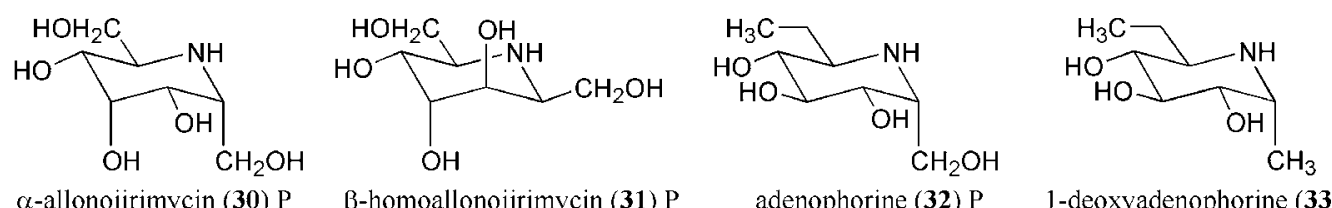

$\alpha$-allonojirimycin (30) $\mathrm{P}$

$\beta$-homoallonojirimycin (31) $\mathrm{P}$

adenophorine (32) $\mathrm{P}$

1-deoxyadenophorine (33) $\mathrm{P}$
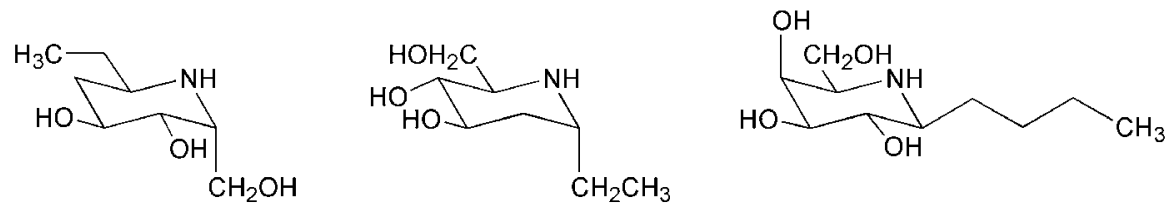

5-deoxyadenophorine (34) P

$\alpha$-1-C-ethylfagomine (35) P

$\beta$-1-C-butyl-1-galactonojirimycin (36) P 
Figure S2. Iminosugars with the same molecular formula as bastzellaside A.
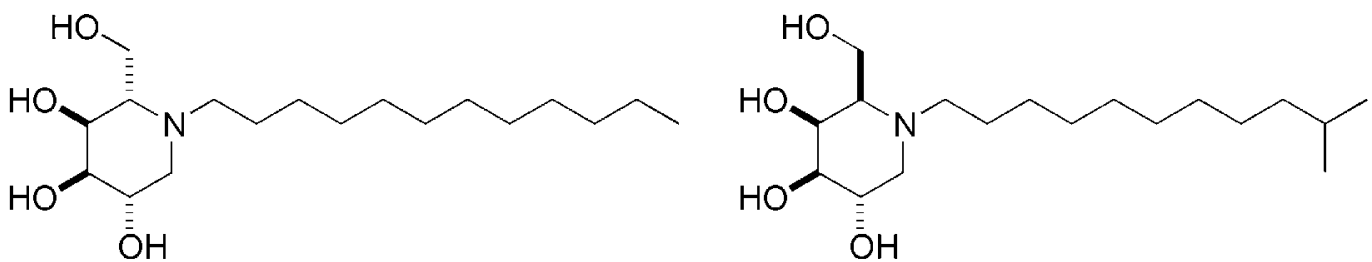

$\mathrm{N}$-dodecyl-5(S)-1-deoxygalactonojirimycin (37) N-10-methyl-undecyl-1-deoxygalactonojirimycin (38)
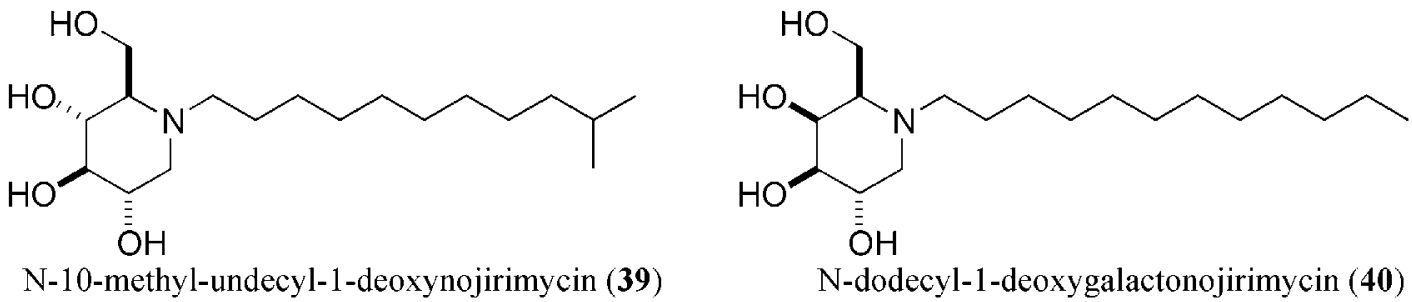

$\mathrm{N}$-10-methyl-undecyl-1-deoxynojirimycin (39)
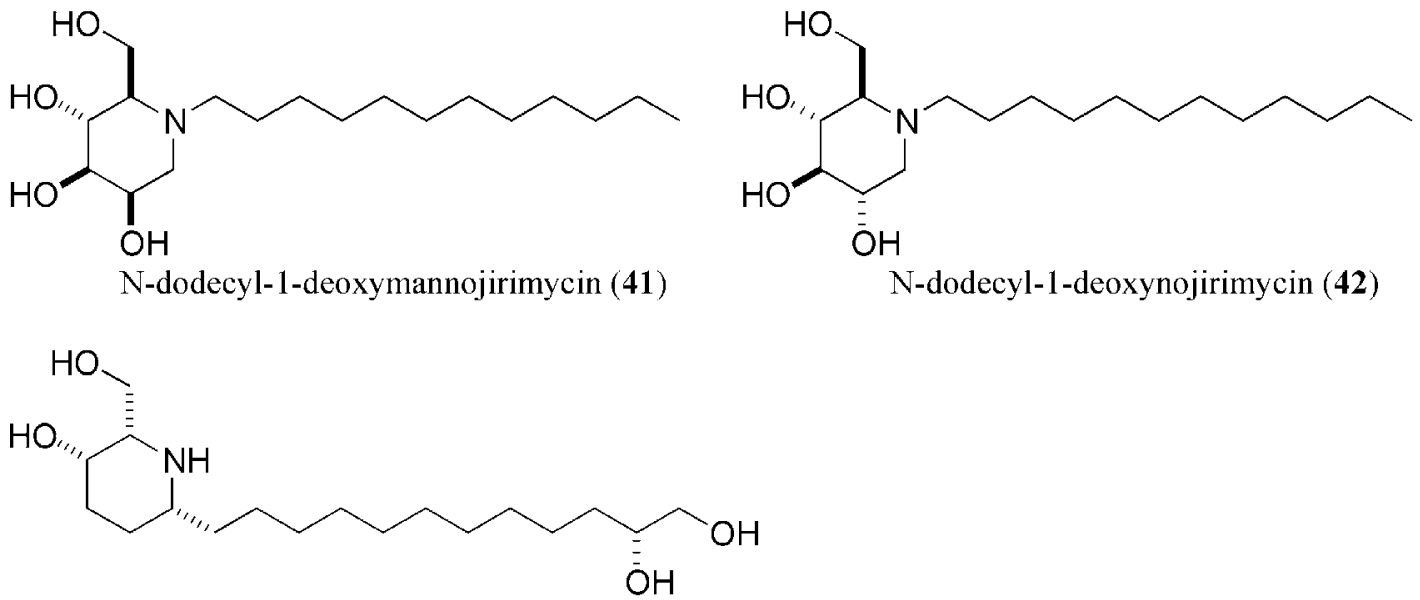

11'(R)-leptophylline A (43) 
Figure S3. Plant-derived alkaloids considered related to batzellasides A-C.

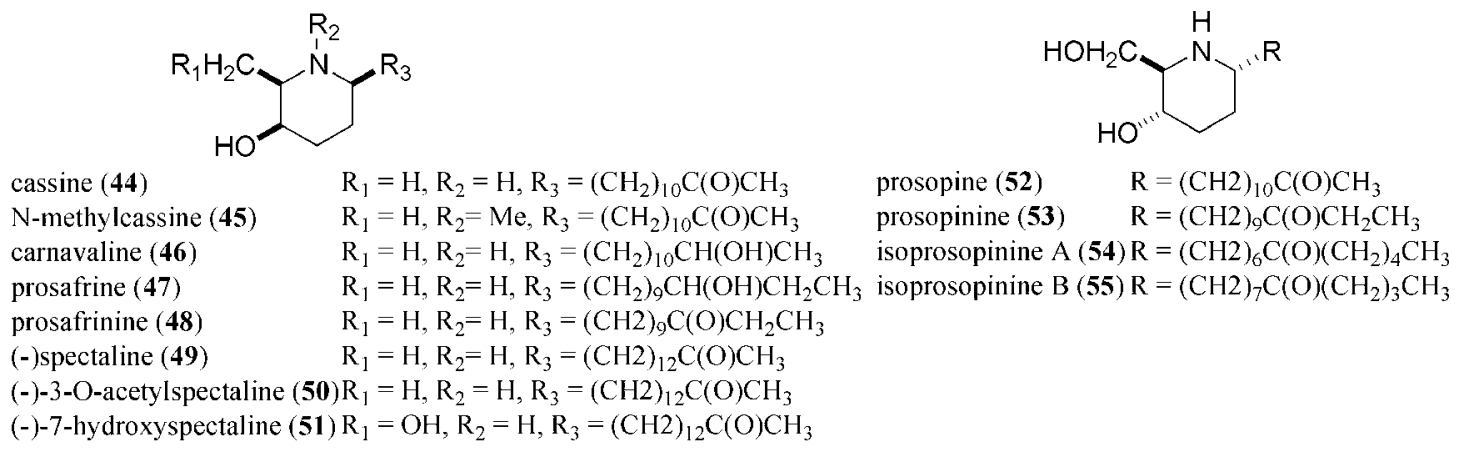
$\mathrm{R}_{1}=\mathrm{H}, \mathrm{R}_{2}=\mathrm{H}, \mathrm{R}_{3}=\left(\mathrm{CH}_{2}\right)_{9} \mathrm{CH}(\mathrm{OH}) \mathrm{CH}_{2} \mathrm{CH}_{3}$ isoprosopinine $\mathrm{B}(\mathbf{5 5}) \mathrm{R}=(\mathrm{CH} 2)_{7} \mathrm{C}(\mathrm{O})\left(\mathrm{CH}_{2}\right)_{3} \mathrm{CH}_{3}$

$\mathrm{R}=(\mathrm{CH} 2)_{10} \mathrm{C}(\mathrm{O}) \mathrm{CH}_{3}$ $\begin{array}{lrl}\text { prosopinine }(\mathbf{5 3}) \quad \mathrm{R} & =(\mathrm{CH} 2)_{9} \mathrm{C}(\mathrm{O}) \mathrm{CH}_{2} \mathrm{CH}_{3} \\ \text { isoprosopinine } \mathrm{A}(\mathbf{5 4}) \mathrm{R} & =(\mathrm{CH} 2)_{6} \mathrm{C}(\mathrm{O})\left(\mathrm{CH}_{2}\right)_{4} \mathrm{CH}_{3}\end{array}$

(1)

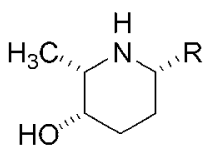

(+)spectaline $(\mathbf{5 6}) \mathrm{R}=\left(\mathrm{CH}_{2}\right)_{12} \mathrm{C}(\mathrm{O}) \mathrm{CH}_{3}$ spectalinine (57) $\mathrm{R}=\left(\mathrm{CH}_{2}\right)_{12} \mathrm{CH}(\mathrm{OH}) \mathrm{CH}_{3}$
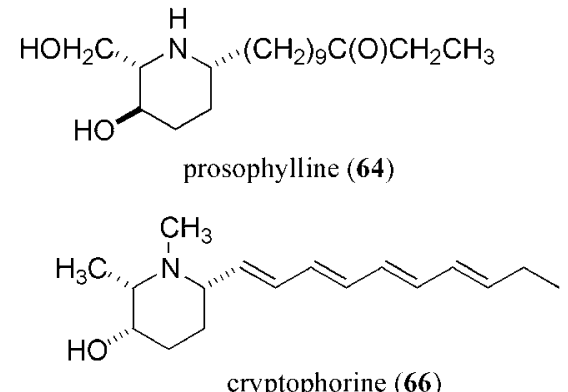

cryptophorine (66)

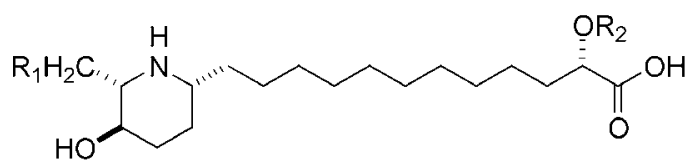

morusimic acid C (68) $\mathrm{R}_{1}=\mathrm{H}, \mathrm{R}_{2}=\mathrm{Glc}$

morusimic acid D (69) $\mathrm{R}_{1}=\mathrm{R}_{2}=\mathrm{H}$

morusimic acid $\mathrm{E}(\mathbf{7 0}) \mathrm{R}_{1}=\mathrm{OH}, \mathrm{R}_{2}=\mathrm{Glc}$<smiles>C[C@@H]1N[C@H](CCCCCCCCO)CC[C@H]1O</smiles><smiles>C[C@H]1CC[C@@H](O)[C@@H](C)N1</smiles>

$$
\begin{aligned}
& \begin{array}{ll}
\text { iso-6-cassine (58) } & \\
\text { iso-6-carnavaline (59) } & \mathrm{R}_{1}=\mathrm{H}, \mathrm{R}_{2}=\left(\mathrm{CH}_{2}\right)_{10} \mathrm{C}(\mathrm{O}) \mathrm{CH}_{3} \\
\text { 2-benoyloxo-iso-6-cassine (60) } \mathrm{R}_{1}=\mathrm{Bn}, \mathrm{R}_{2}=\left(\mathrm{CH}_{2}\right)_{10} \mathrm{C}(\mathrm{O}) \mathrm{CH}_{3} \\
\text { iso-6-spectaline (61) } & \mathrm{R}_{1}=\mathrm{H}, \mathrm{R}_{2}=\left(\mathrm{CH}_{2}\right)_{12} \mathrm{C}(\mathrm{O}) \mathrm{CH}_{3} \\
\text { spectamine } \mathrm{A}(\mathbf{6 2}) & \mathrm{R}_{1}=\mathrm{C}(\mathrm{O}) \mathrm{Ph}_{2}, \mathrm{R}_{2}=\left(\mathrm{CH}_{2}\right)_{10} \mathrm{C}(\mathrm{O}) \mathrm{CH}_{3} \\
\text { spectamine B (63) } & \mathrm{R}_{1}=\mathrm{C}(\mathrm{O}) \mathrm{CH}_{3}, \mathrm{R}_{2}=\left(\mathrm{CH}_{2}\right)_{10} \mathrm{C}(\mathrm{O}) \mathrm{CH}_{3}
\end{array}
\end{aligned}
$$

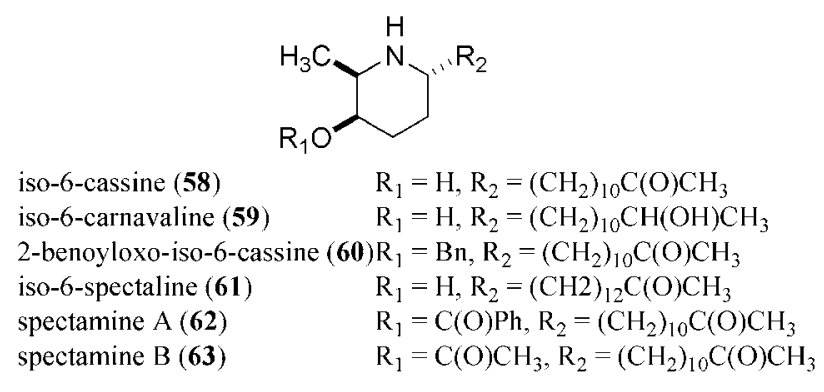

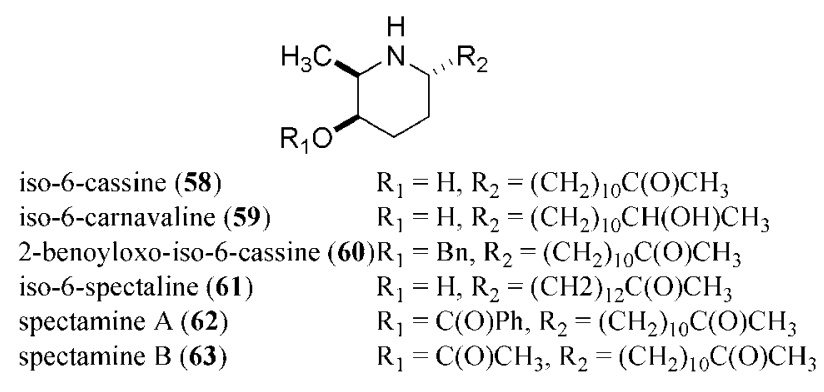

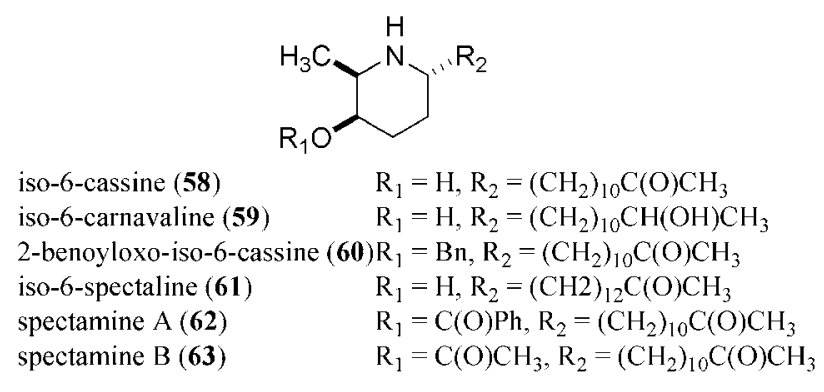

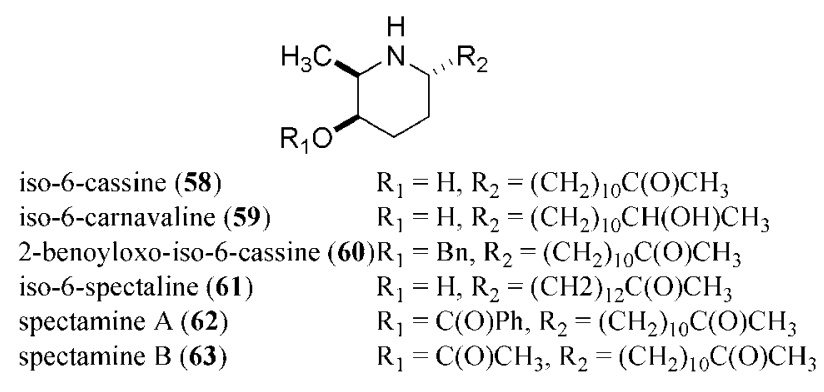$$
\begin{array}{ll}
\text { iso-6-cassine (58) } & \mathrm{R}_{1}=\mathrm{H}, \mathrm{R}_{2}=\left(\mathrm{CH}_{2}\right)_{10} \mathrm{C}(\mathrm{O}) \mathrm{CH}_{3} \\
\text { iso-6-carnavaline (59) } & \mathrm{R}_{1}=\mathrm{H}, \mathrm{R}_{2}=\left(\mathrm{CH}_{2}\right)_{10} \mathrm{CH}(\mathrm{OH}) \mathrm{CH}_{3} \\
\text { 2-benoyloxo-iso-6-cassine (60) } \mathrm{R}_{1}=\mathrm{Bn}, \mathrm{R}_{2}=\left(\mathrm{CH}_{2}\right)_{10} \mathrm{C}(\mathrm{O}) \mathrm{CH}_{3} \\
\text { iso-6-spectaline (61) } & \mathrm{R}_{1}=\mathrm{H}, \mathrm{R}_{2}=(\mathrm{CH})_{12} \mathrm{C}(\mathrm{O}) \mathrm{CH}_{3} \\
\text { spectamine } \mathrm{A}(\mathbf{6 2}) & \mathrm{R}_{1}=\mathrm{C}(\mathrm{O}) \mathrm{Ph}_{2}, \mathrm{R}_{2}=\left(\mathrm{CH}_{2}\right)_{10} \mathrm{C}(\mathrm{O}) \mathrm{CH}_{3} \\
\text { spectamine B (63) } & \mathrm{R}_{1}=\mathrm{C}(\mathrm{O}) \mathrm{CH}_{3}, \mathrm{R}_{2}=\left(\mathrm{CH}_{2}\right)_{10} \mathrm{C}(\mathrm{O}) \mathrm{CH}_{3}
\end{array}
$$
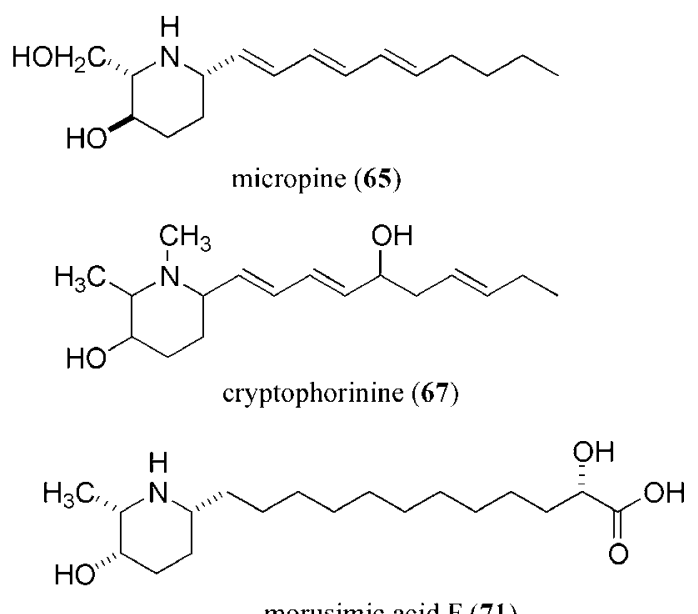

morusimic acid F (71)

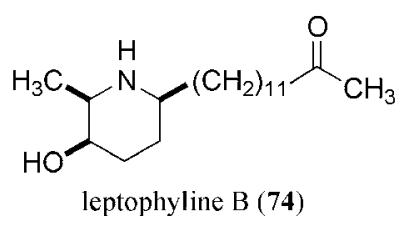


Figure S4. Marine natural products related to batzellasides A-C.

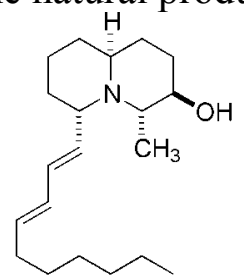

clavepictine A ( 75 )

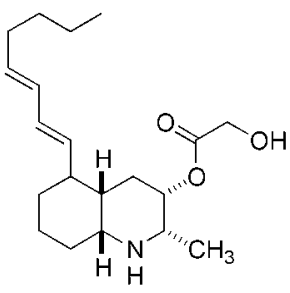

lepadin A (78)

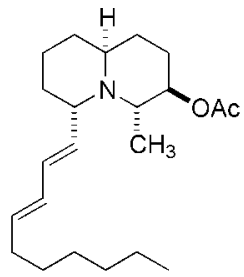

clavepictine B (76)

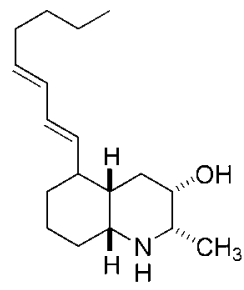

lepadin B (79)

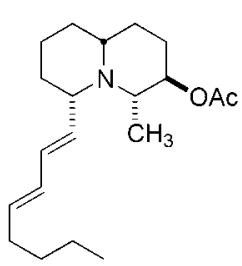

pictamine (77)

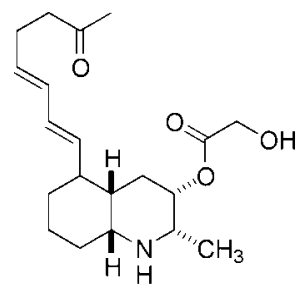

lepadin $\mathrm{C}(\mathbf{8 0})$

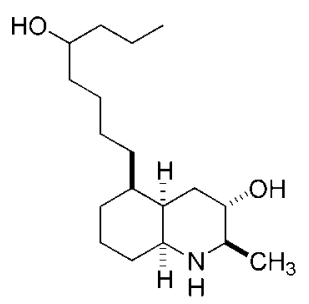

lepadin D (81)

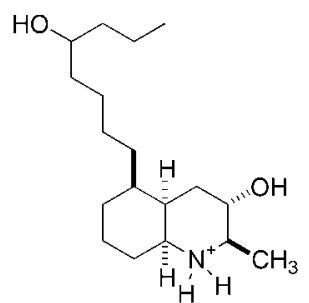

lepadin D quaternary nitrogen dervative (82)

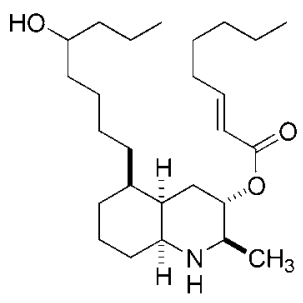

lepadin E (83)

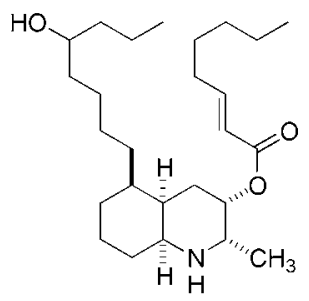

(-) lepadin F (84)

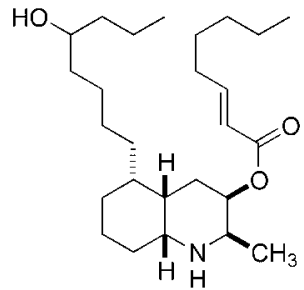

(+) lepadin F (74)

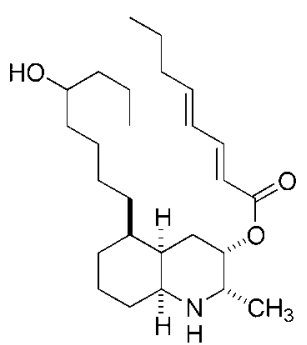

lepadin G (75)

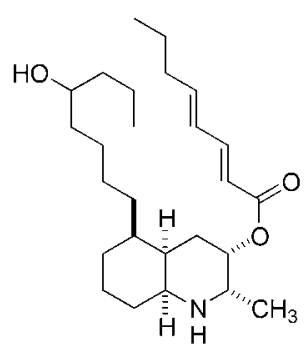

lepadin H (76) 


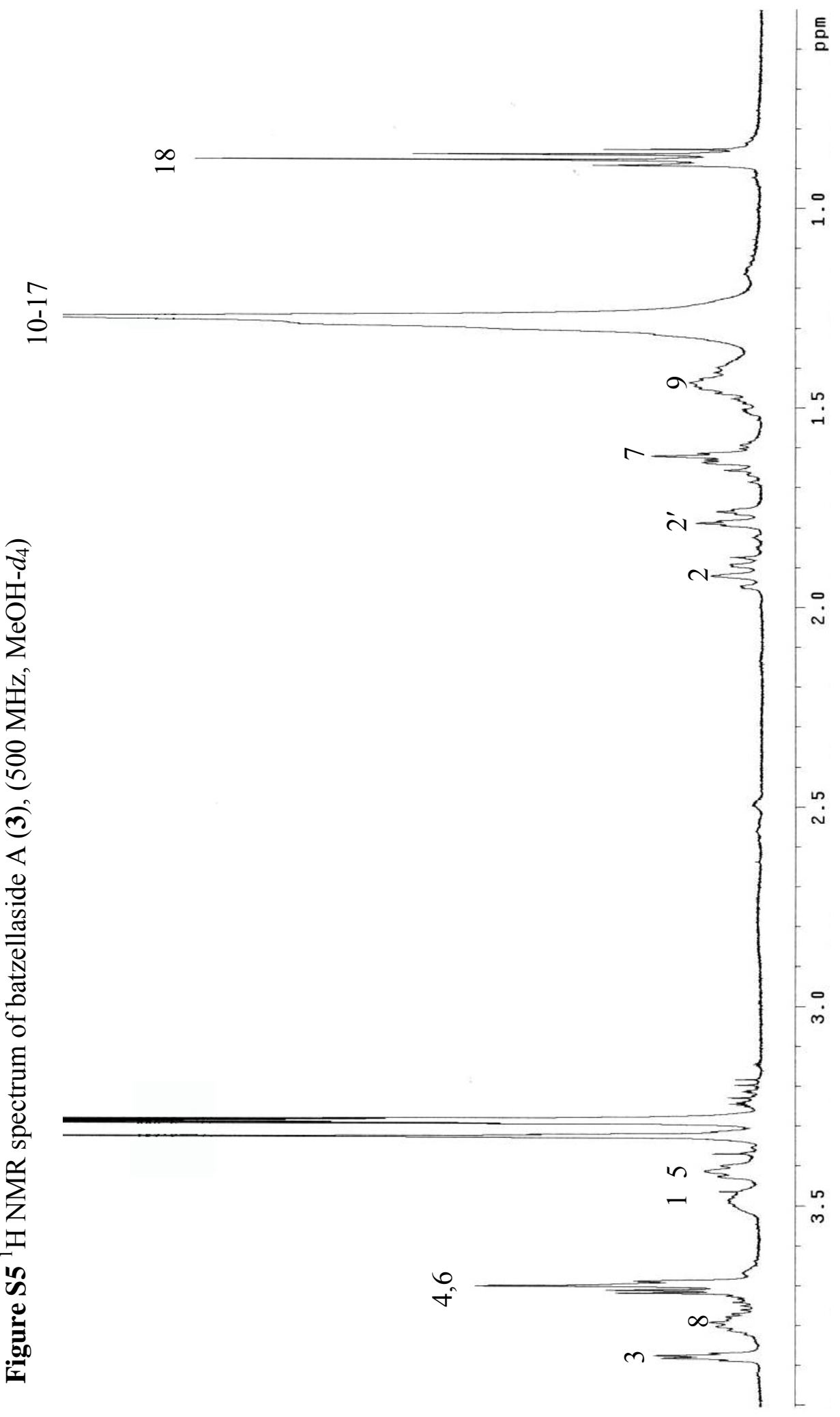




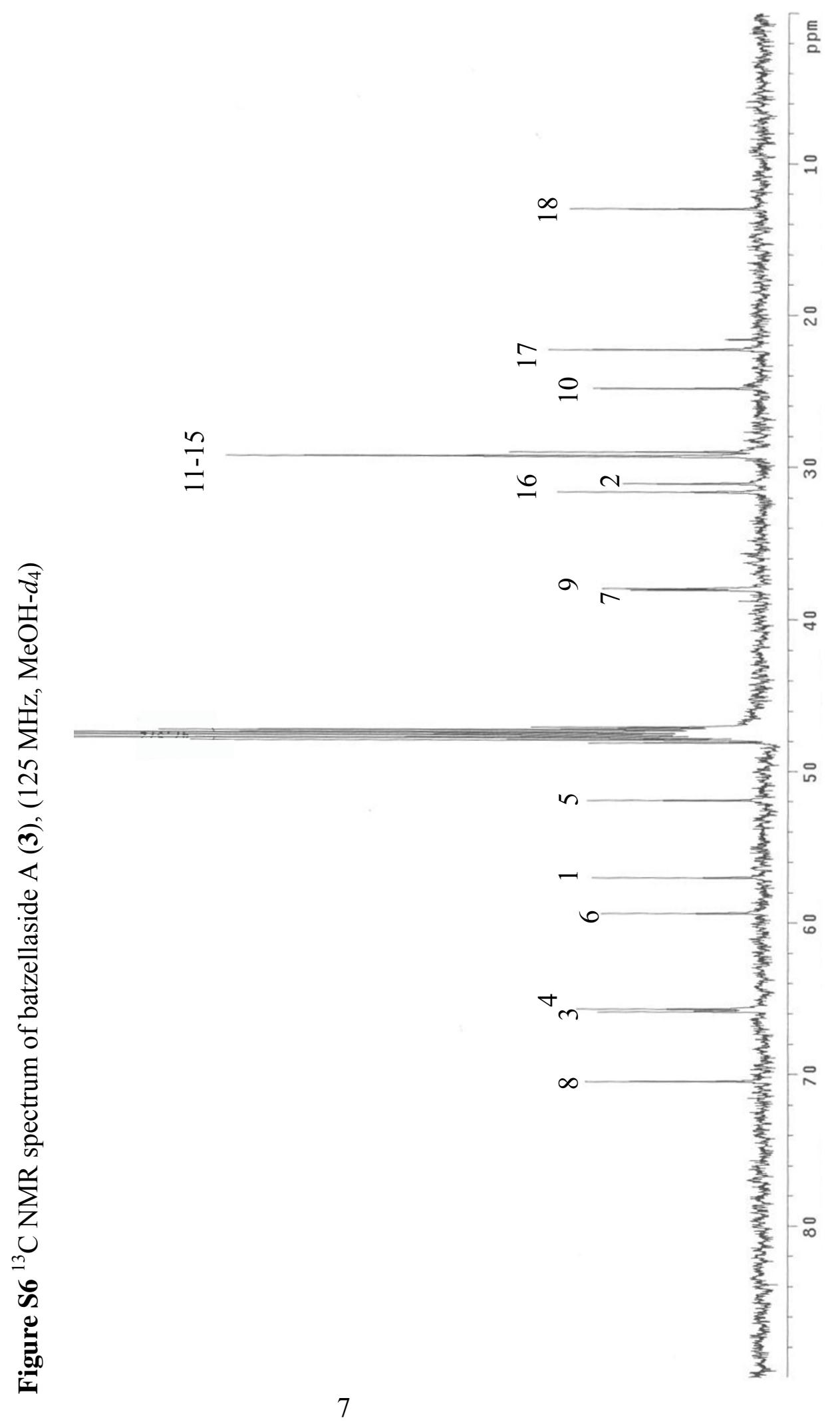




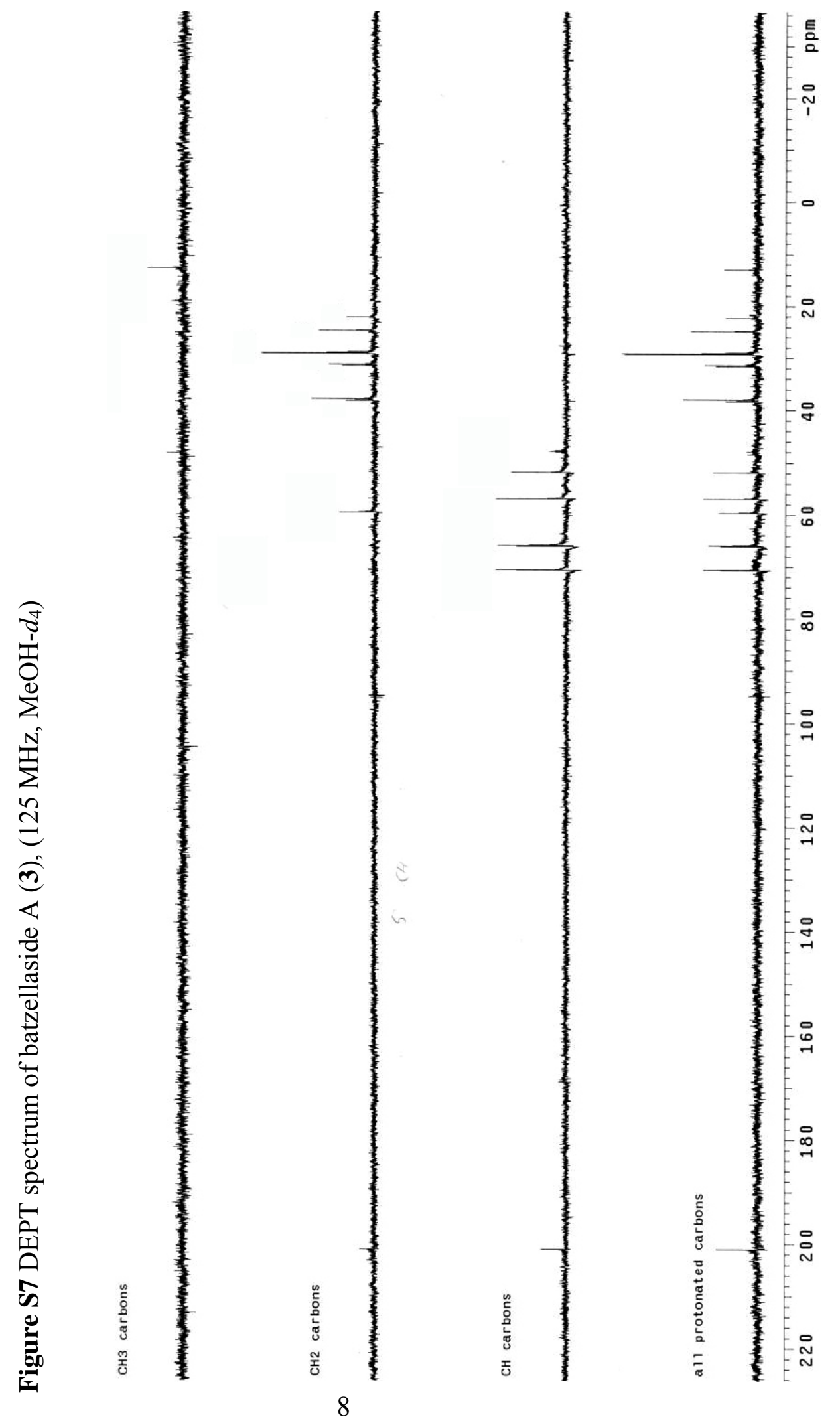


Figure S8 ${ }^{1} \mathrm{H}{ }^{1} \mathrm{H}$ COSY spectrum of batzellaside A (3), (500 MHz, MeOH- $\left.d_{4}\right)$

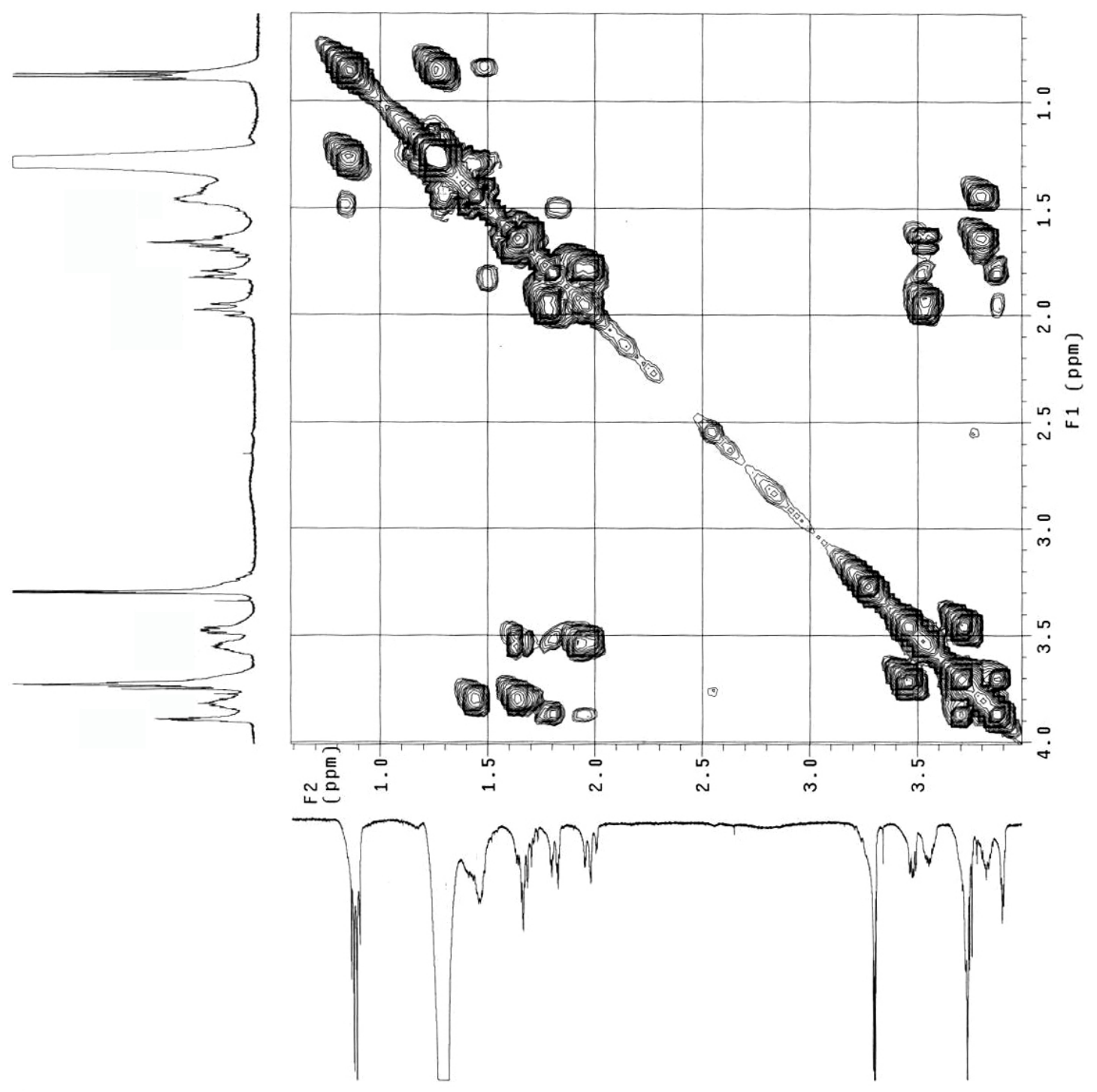


Figure S9 gHMQC spectrum of batzellaside A (3), (500 MHz, MeOH-d $)$

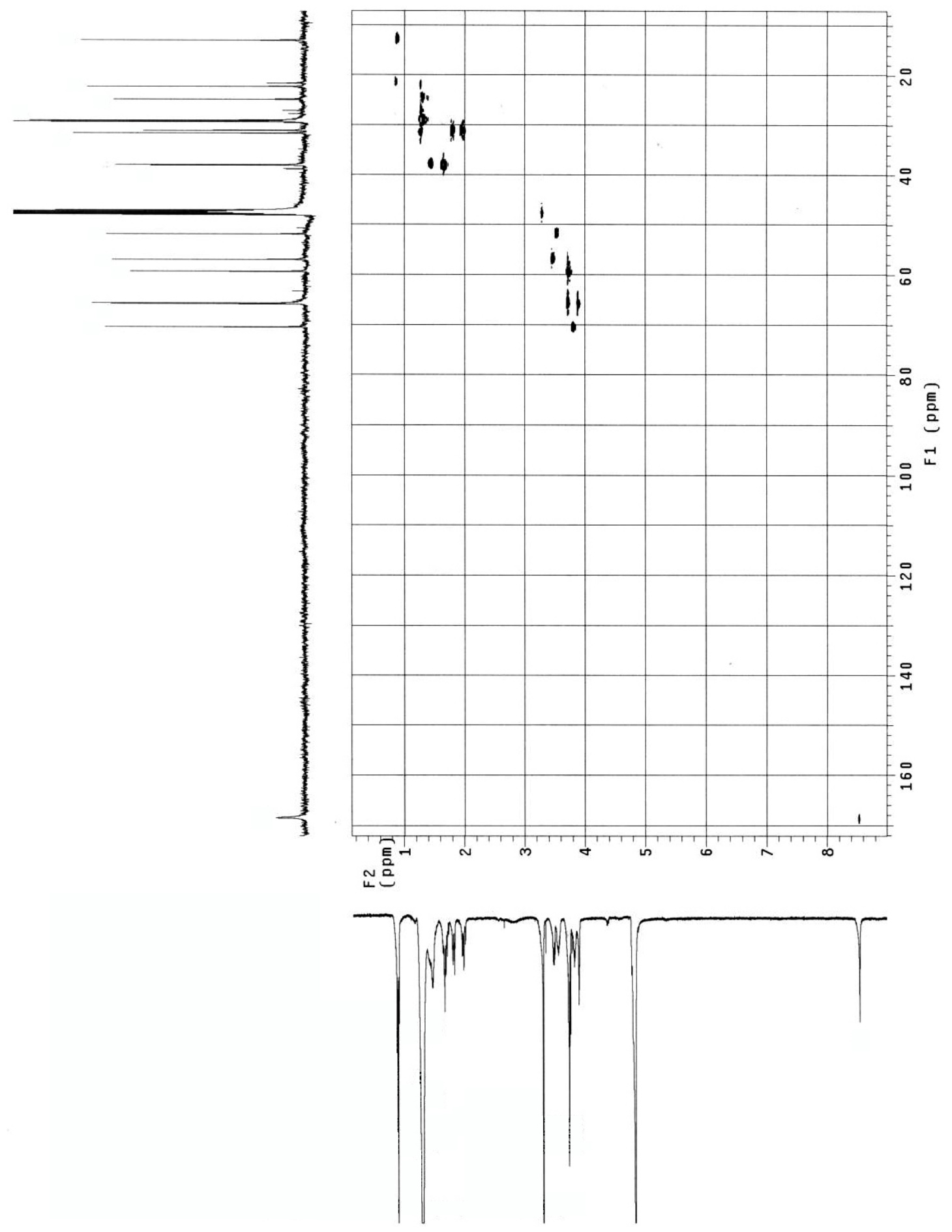


Figure S10 $\mathrm{gHMBC}(\mathrm{J}=8 \mathrm{~Hz})$ spectrum of batzellaside A (3), (500 MHz, MeOH- $\left.d_{4}\right)$

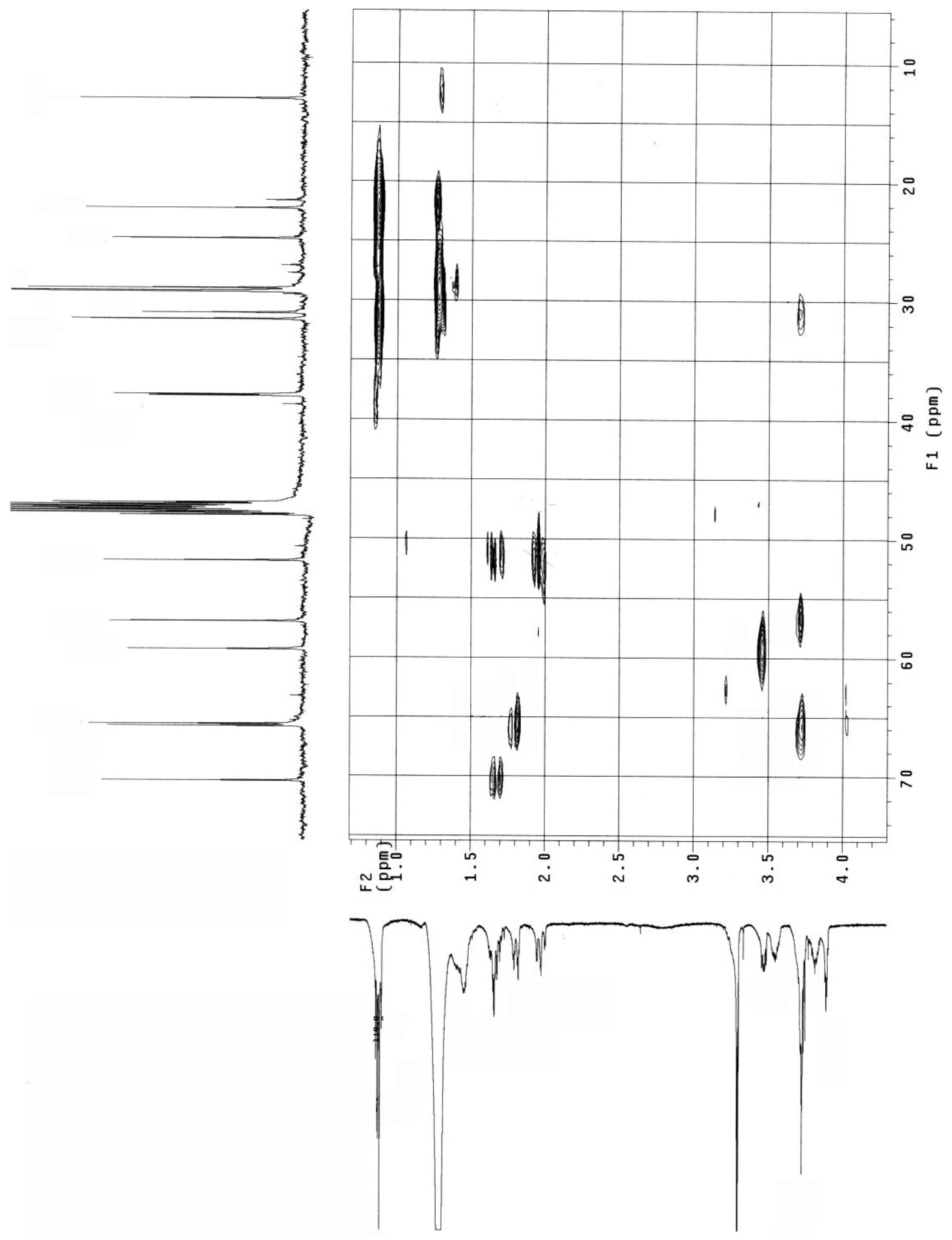


Figure S11 gHMBC ( $\mathrm{g}=4 \mathrm{~Hz})$ spectrum of batzellaside A (3), (500 MHz, MeOH- $\left.d_{4}\right)$

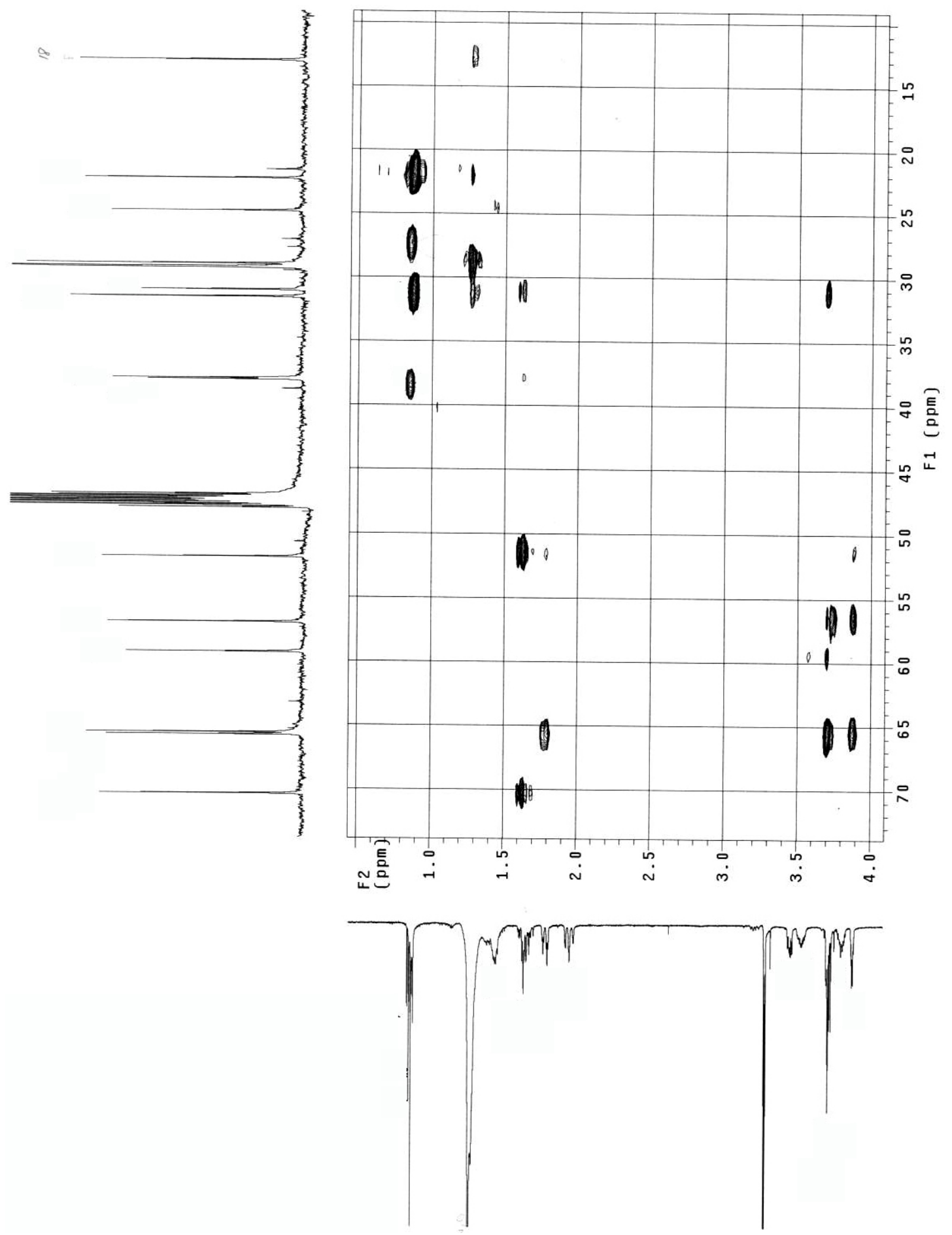


Figure S12 ${ }^{1} \mathrm{H}{ }^{1} \mathrm{H}$ NOESY spectrum of batzellaside A (3), $\left(500 \mathrm{MHz}, \mathrm{MeOH}-d_{4}\right)$

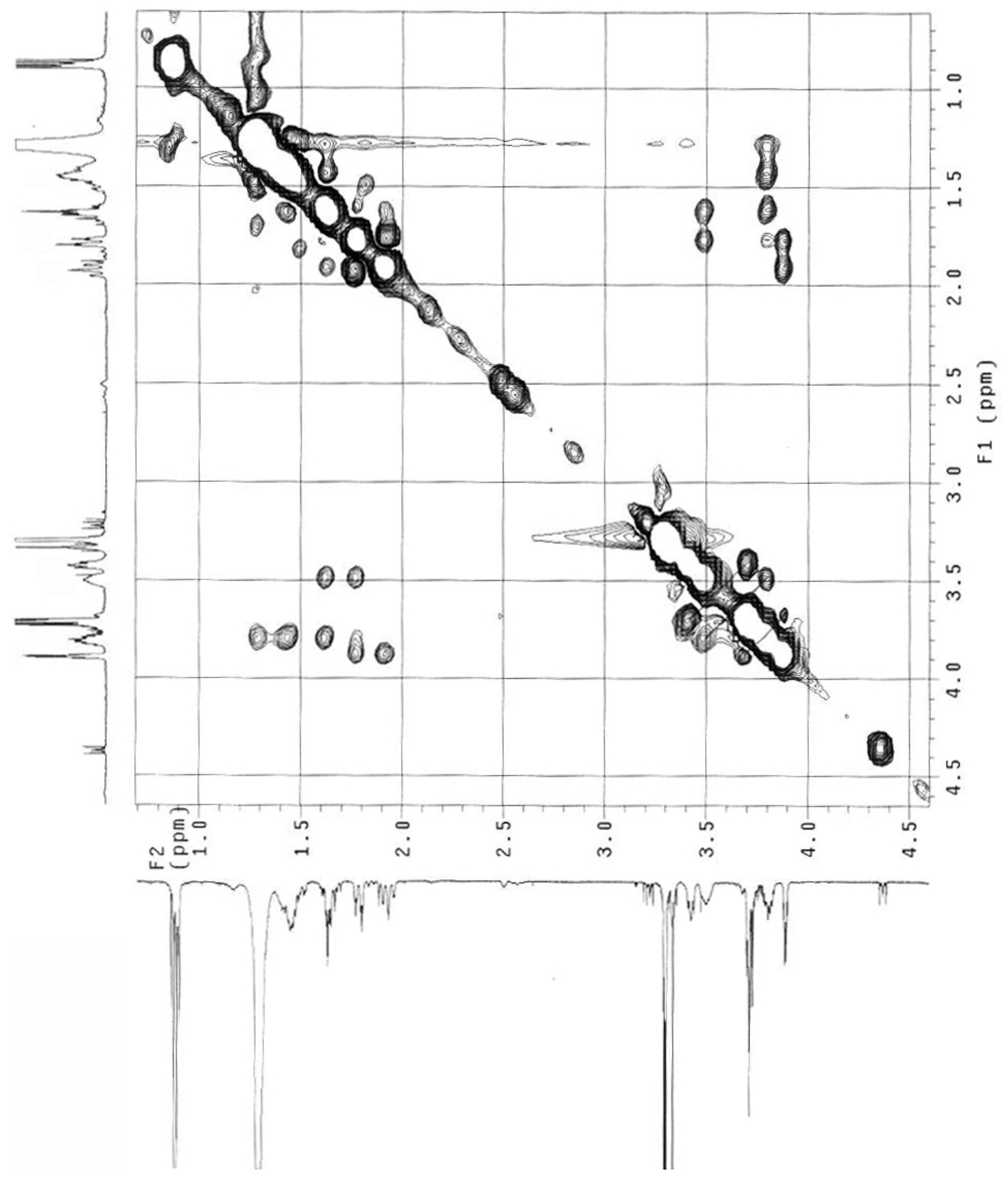




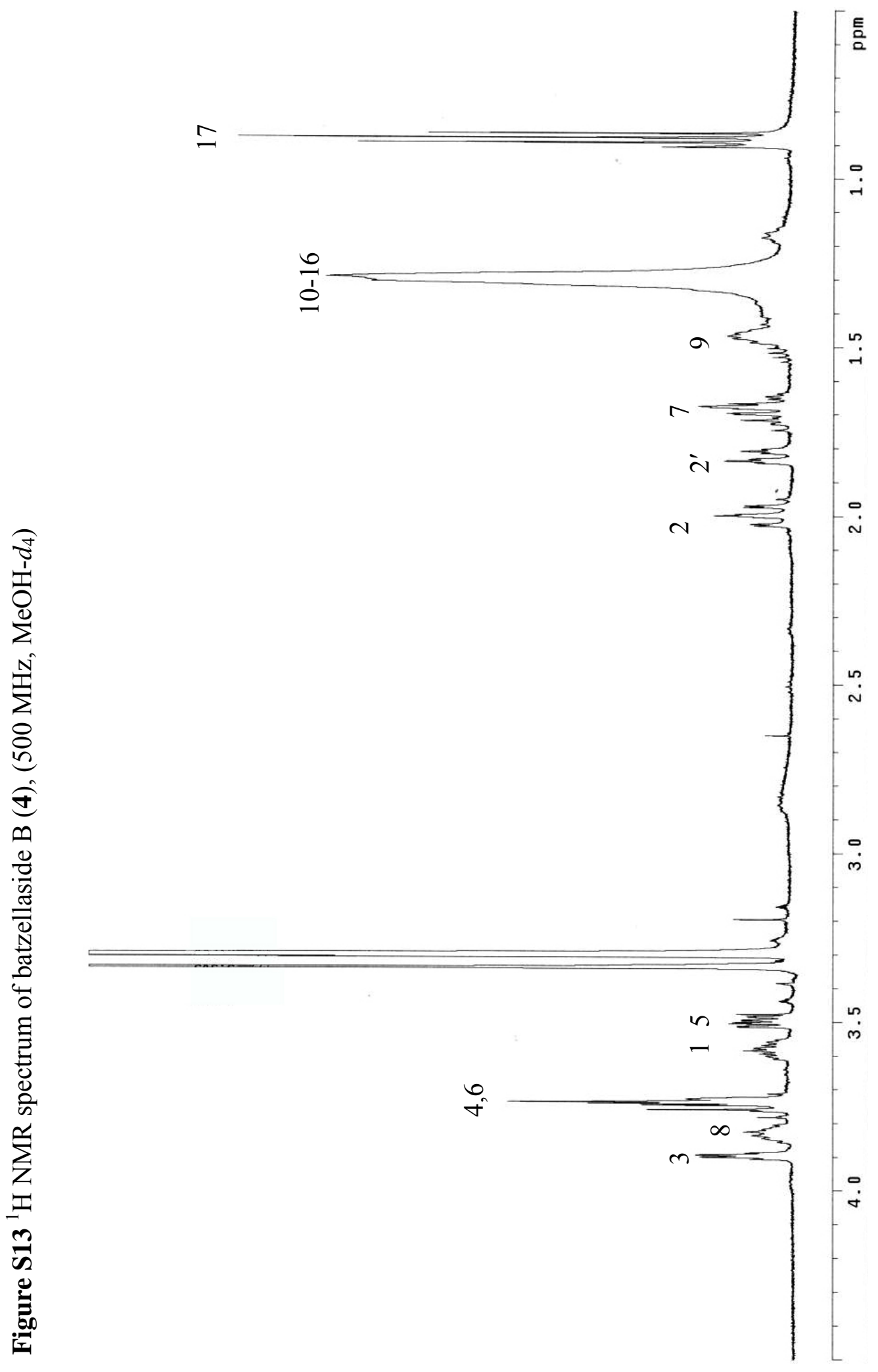




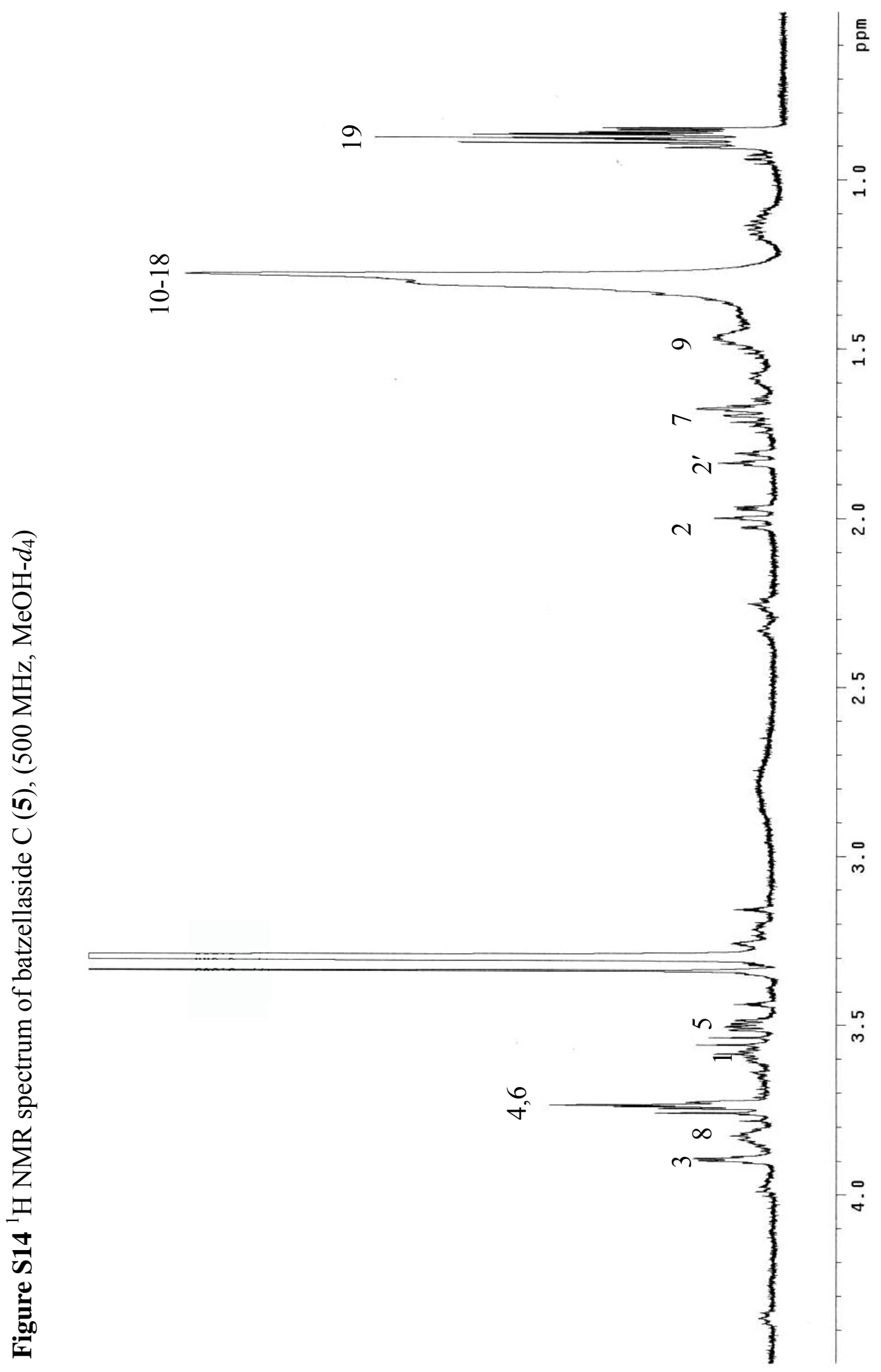


Figure S15. Above Water Photograph of 00216.

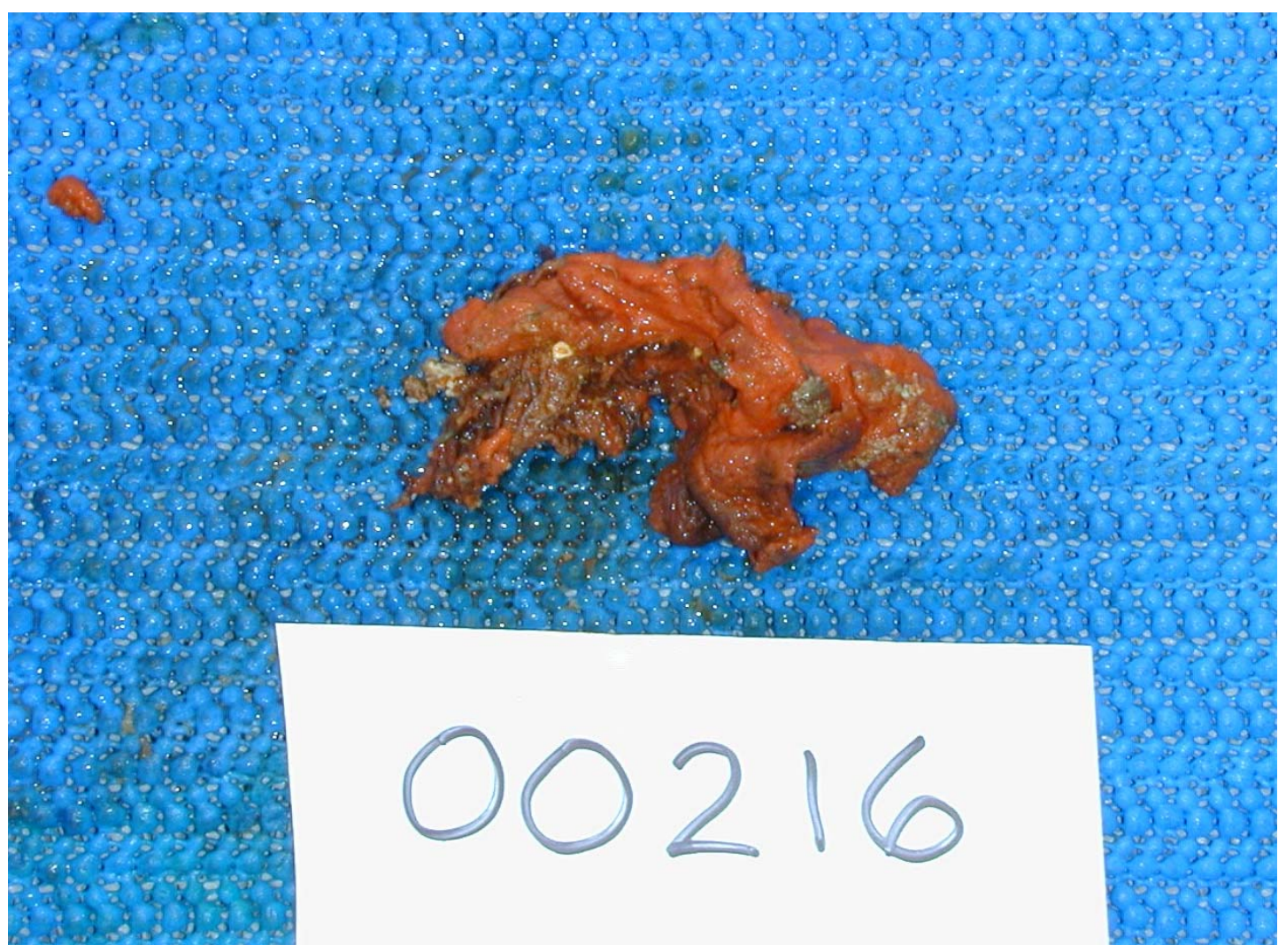


Figure S16. Outline of isolation of halitoxin and batzellasides A-C.

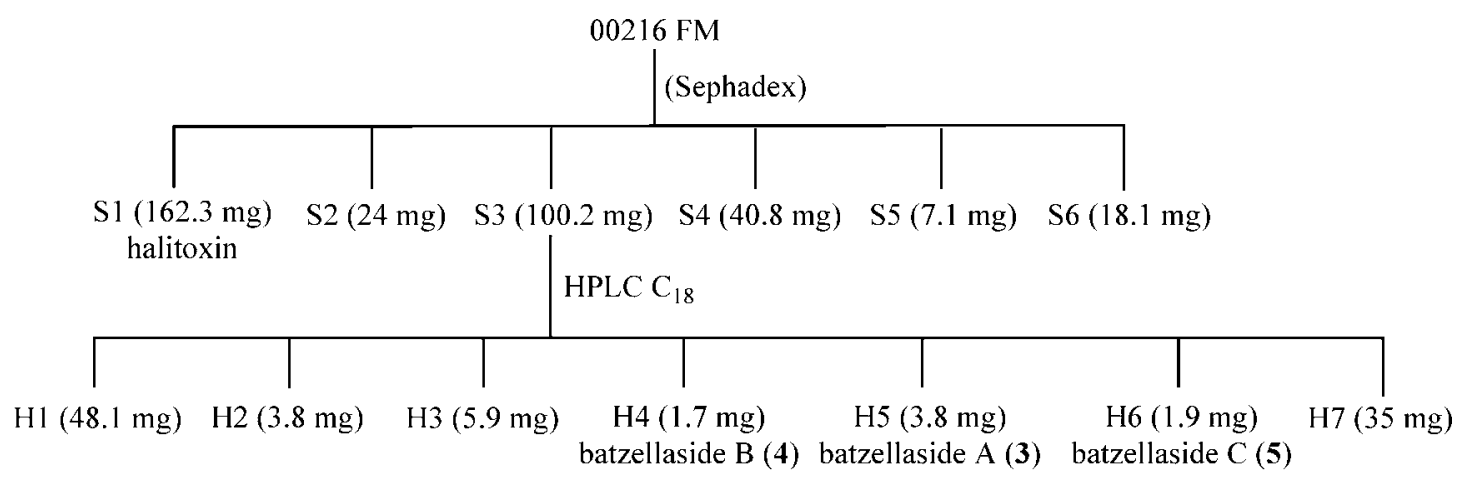

